\title{
High frequency relaxation in nematic liquid crystal mixtures by Brillouin scattering
}

\author{
Authors: I.G. Siny, C.-S. Tu, and V. Hugo Schmidt
}

This is an Accepted Manuscript of an article published in Molecular Crystals and Liquid Crystals Science and Technology. Section A. Molecular Crystals and Liquid Crystals in 1995, available online: http://www.tandfonline.com/10.1080/10587259508037326.

I.G. Siny, C.-S. Tu, and V.H. Schmidt, "High frequency relaxation in nematic liquid crystal mixtures by Brillouin scattering," Molecular Crystals and Liquid Crystals Science and Technology. Section A. Molecular Crystals and Liquid Crystals 269, 125-132 (1995).

Made available through Montana State University's ScholarWorks scholarworks.montana.edu 


\title{
High Frequency Relaxation in Nematic Liquid Crystal Mixtures by Brillouin Scattering
}

\author{
I. G. SINY,* C. -S. TU and V. H. SCHMIDT \\ Department of Physics, Montana State University Bozeman, Montana 59717
}

(Received October 25, 1994)

\begin{abstract}
Brillouin scattering as a function of scattering angles from $180^{\circ}$ to $\sim 10^{\circ}$ has been measured in the nematic phases of two liquid crystal mixtures, E. Merck ZLI-2861 and E7, without any preferred orientation. Such measurements at room temperature cover the frequency range from $\sim 10$ to $\sim 1 \mathrm{GHz}$. The behavior of both the frequency shifts and linewidths is explained by a single time internal relaxation process with the characteristic frequencies 3.2 (ZLI-2861) and 1.2 (E7) GHz respectively. The behavior of the hypersonic attenuation shows no correlation with the static bulk viscosity in the nematic phase.
\end{abstract}

Keywords: Liquid crystal, nematic, relaxation, Brillouin scattering

\section{INTRODUCTION}

After the first wave of interest in Brillouin scattering measurements in liquid crystals in the 1970's, the number of publications reduced considerably. The response of liquid crystals at high frequencies characteristic of the Brillouin scattering method was not strong enough to reveal specific acoustic anomalies near phase transformations. The usual relaxor system exhibited by most materials appeared to be clamped at high frequencies and no coupling of the hypersonic phonons to liquid crystal order was observed. This situation can be illustrated by results for MBBA, which will contrast with our more interesting results for two liquid crystal mixtures.

The previous results for MBBA are different from those for some other liquid crystals where contradictory data had been found. ${ }^{1,2}$ The Brillouin spectra of pure MBBA ${ }^{3-5}$ and an MBBA-EBBA mixture ${ }^{6}$ obtained in different laboratories have not shown any significant anomalies at the isotropic-nematic transformation, whereas ultrasonic measurements indicated anomalous behavior of dynamical properties near the phase transition. ${ }^{7-8}$ Comparison of two sets of data ${ }^{5-6}$ pointed out the existence of strong dispersion for sound velocity and damping. However, it seems that behavior of MBBA can not be completely described by a single relaxation process at ultrasonic frequencies even if we exclude from consideration the temperature region near the transition where strong evidence of multiple relaxation was found. ${ }^{8}$ Brillouin measurements show

*Present address: A. F. Ioffe Physical Technical Institute, Russian Academy of Sciences, St. Petersburg 194021, Russia. 
evidence for a new internal relaxation process at $\sim 2.5 \mathrm{GHz}^{5}$ or $\sim 2 \mathrm{GHz}^{9}$ This situation seems normal for such complicated systems as liquid crystals because one can expect different relaxations to contribute to the dispersion of both sound velocity and attenuation in different frequency regions. The assignment of attenuation to particular intramolecuar perturbations is difficult. In this connection one can note the attempt to reveal the rotational isomerism contribution in the nematic phase of Licristal Merck IV which has flexible ends of the mesogen, very similar to the structure of MBBA. ${ }^{10}$

The frequency region of Brillouin scattering measurements is very important for a complete study in an extended frequency range, especially for liquid crystals with low viscosity for which we expect to find dispersion just in the Brillouin scattering range. Such materials are important because of the modern tendency to create complex mixtures to reduce the liquid crystal viscosity. For this reason we chose for our Brillouin scattering measurements two liquid crystal mixtures, E. Merck ZLI-2861 and E7, with low but slightly different bulk viscosities. Both mixtures have high clearing temperatures, $T_{c l}$, and broad regions of the nematic phase in the intervals $233 \mathrm{~K}<T<T_{c l}=367 \mathrm{~K}$ for ZLI-2861 and $T<T_{c l}=334 \mathrm{~K}$ for E7. Therefore we expect that additional attenuation due to critical phenomena near phase transitions does not contribute appreciably in our measurements at room temperature.

\section{EXPERIMENTAL DETAILS}

According to the information available from E. Merck Industries, E7 is a mixture of cyanobiphenyls and cyanoterphenyls with density $\sim 0.98 \mathrm{~g} / \mathrm{cm}^{3}$ (at room temperature) which contains K15, K21, M24 and T15. The ZLI-2861 mixture contains ten compounds with different $n$-alkyl chains containing up to five carbon atoms and its density is about $0.97 \mathrm{~g} / \mathrm{cm}^{3}$.

The frame of an optical cell with two parallel windows (sample thickness $\sim 1.2 \mathrm{~mm}$ ) was made of a massive copper plate to prevent sample heating by the laser beam. This cell was used for scattering at various angles from backscattering to nearly forward scattering. Only $90^{\circ}$-geometry was measured in another cell with two windows at right angles. All our measurements were made in samples of E7 and ZLI-2861 without any preferred orientation. The sample thickness of about $1 \mathrm{~mm}$ was too large to expect a fully oriented state. However, some samples appeared inhomogeneous in polarized light. Therefore we believe that the spread of some experimental points is connected with local orientational effects.

The scattering spectra were excited by an argon laser, $\lambda=514.5 \mathrm{~nm}$. In order to avoid heating the sample, the laser power did not exceed $50 \mathrm{~mW}$ and the laser beam was slightly defocused. The appearance of special speckle patterns was attributed to nonlinear effects but not to heating.

In our experiments the scattering geometry is $x(z, u) x$. Here, " $u$ " means that the collection was not polarization discriminated. The scattered light was analyzed with a five-pass planar Fabry-Perot interferometer and a narrow-band ( $1 \AA$ ) interference filter was used in this part of the optical scheme. The shifts of the Brillouin doublet from the laser line and the linewidth of the Brillouin components at half maximum were measured. To eliminate instrumental broadening and the effect of finite aperture on the 
linewidth and position of the Brillouin doublet, we calibrated our Fabry-Perot interferometer by measuring the Brillouin spectra of fused quartz near the same scattering angles. The results (see Figure 1a) show absence of hypersonic velocity dispersion and a constant value for $\alpha / f^{2}$ (the hypersonic damping related to square of frequency) in the frequency range of our measurements, as expected from extrapolation of published results for fused quartz. ${ }^{11}$ From this we conclude that the effect of finite aperture on the Brillouin component position is negligibly small. The Brillouin line broadening $\delta v_{\text {ang }}$ due to the same effect of finite aperture or collection angle $\delta \theta_{\text {ap }}$ is described by a well-known formula ${ }^{12}$ which to second order in $\delta \theta$ is

$$
\frac{\delta v_{\text {ang }}}{\Delta v}=\frac{\cot \left(\frac{\theta}{2}\right) \delta \theta_{\text {ap }}}{2}-\frac{\left(\delta \theta_{\text {ap }}\right)^{2}}{8}
$$

where $\theta$ is the scattering angle and $\Delta v$ is the Brillouin shift. We found $\delta \theta_{\mathrm{ap}} \sim 0.057$ radians from measurements of fused quart $z$ and even for this small value the broadening problem is still severe in the near-forward scattering for which $\cot (\theta / 2)$ is large. The linewidth of the Rayleigh line in fused quartz was taken as the instrumental broadening $\delta v_{\text {ins. }}$. Then the Brillouin doublet linewidth $\delta v_{\mathrm{ph}}$ at half maximum associated with the amplitude attenuation coefficient $\alpha$ for hypersonic phonons by the simple relation ${ }^{13}$

$$
\delta v_{\mathrm{ph}}=\frac{\alpha V}{\pi}
$$

can be corrected for aperture and instrumental broadening by ${ }^{14}$

$$
\delta v_{\mathrm{ph}}=\left[\left(\delta v_{\mathrm{obs}}\right)^{2}-\left(\delta v_{\mathrm{ang}}\right)^{2}\right]^{1 / 2}-\delta v_{\mathrm{ins}}
$$

where $V$ and $\delta v_{\mathrm{obs}}$ represent the sound velocity and observed linewidth in the Brillouin spectra. Using Equations 1-3 we can calculate the ratio of the hypersonic attenuation to the square of frequency, $\alpha /(\Delta v)^{2}$, for ZLI-2861 and E7 as functions of the scattering angle (or hypersonic phonon frequency). Both scales are shown at the top and bottom respectively, in Figures 1 and 2.

\section{RESULTS AND DISCUSSION}

As one can see in Figures $1 \mathrm{~b}$ and 2a, both ZLI-2861 and E7 show a significant increase of $\alpha / f^{2}$ (here $f \equiv \Delta v$ ) as frequency decreases, in contrast to fused quartz (see Figure 1a). This effect was follwed over a decade of frequency range which is usually not enough to encompass fully even a single relaxation process in fluids. Unfortunately, we have not found any results of ultrasonic measurement for E7 and ZLI-2861. Thus, we have to make qualitative conclusions based on the Brillouin scattering data.

It seems that the behavior of the quantity $\alpha / f^{2}$ is different in these two liquid crystals. The initial points from backscattering are very close for both fluids, but a difference is 

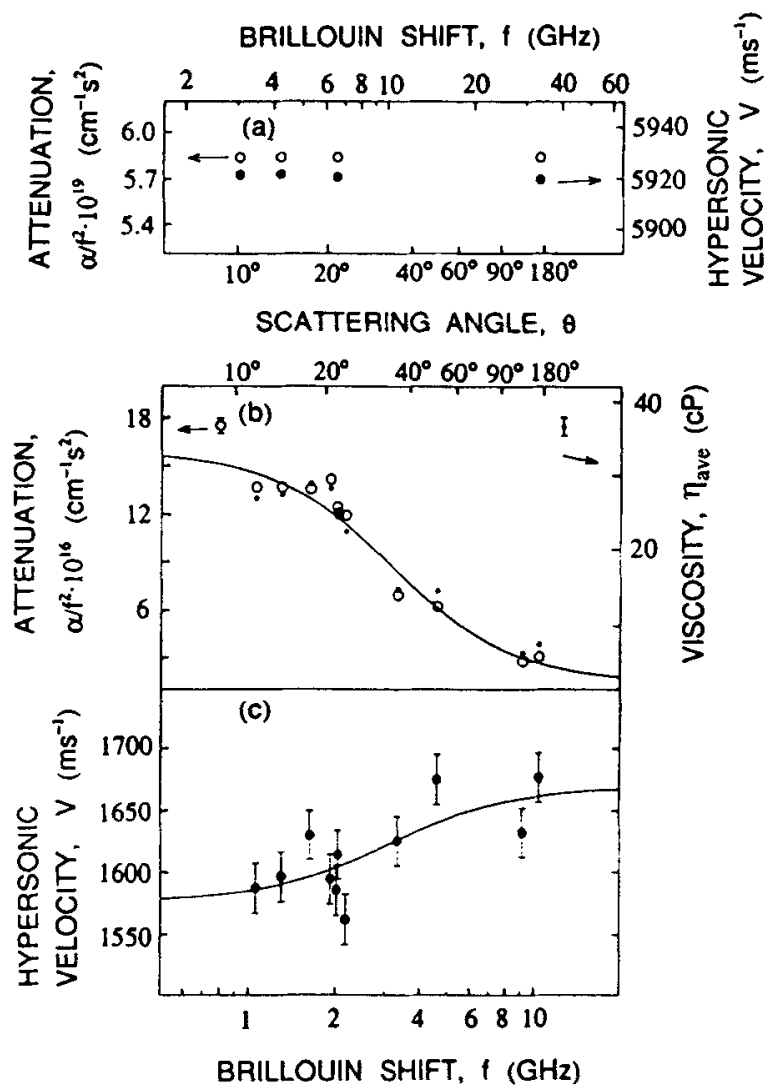

FIGURE 1 (a) Hypersonic velocity (๑) and sound attenuation $\alpha / f^{2}(O)$ vs. frequency for fused quartz. (b) Sound attenuation $\alpha / f^{2}(O)$ and viscosity $\eta_{\text {sue }}(\bullet)$ vs. frequency for ZLI-2861. Solid line is the fit by Equation (4). (c) Hypersonic velocity vs. frequency for ZLI-2861. Solid line is the fit by Equation (5).

seen at lower frequencies. The points for ZLI-2861 from nearly forward scattering tend toward saturation, but this does not occur for E7 although the values of $\alpha / f^{2}$ in both E7 and ZLI-2861 are close for forward scattering. Doubtlessly, internal relaxation processes exist in the hypersonic region in both liquid crystals. The experimental data satisfactorily fit the curves calculated from the well-known relationship for a single relaxation time process ${ }^{15}$

$$
\left.\frac{\alpha}{f^{2}}=\frac{A}{\left[1+\left(\frac{f}{f_{r}}\right)^{2}\right.}\right]^{+B}
$$

where $A$ and $B$ are constants and $f_{r}$ is the relaxation frequency. In accordance with the qualitative consideration above, by fitting the experimental points in Figures $1 \mathrm{~b}$ and $2 \mathrm{a}$ with Equation (4), we obtained $f_{r}=3.2 \mathrm{GHz}, A=14.55 \mathrm{~cm}^{-1} \mathrm{~s}^{2}$ and $B=1.437 \mathrm{~cm}^{-1} \mathrm{~s}^{2}$ 


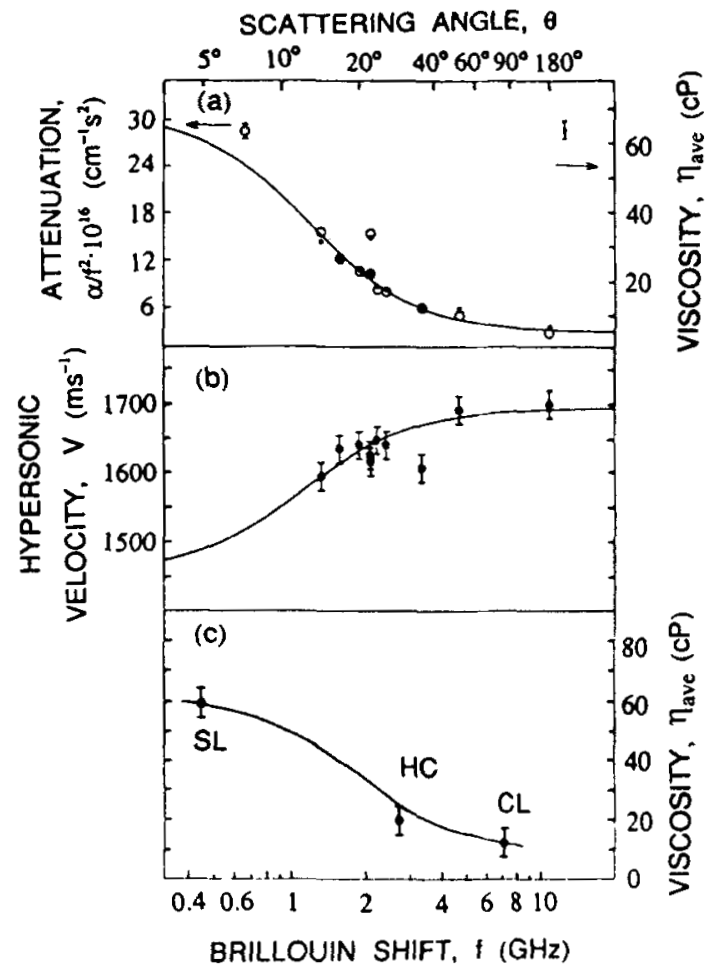

FIGURE 2 (a) Sound attenuation $\alpha / f^{2}(O)$ and viscosity $\eta_{\text {ave }}(\bullet)$ vs. frequency for E7. Solid line is the fit by Equation (4). (b) Hypersonic velocity vs. frequency for E7. Solid line is the fit by Equation (5). (c) Viscosity $\eta_{\text {ave }}$ vs. frequency for MBBA [9]. Solid line is the fit to a single relaxation time model.

for ZLI-2861, and $f_{r}=1.2 \mathrm{GHz}, A=28.35 \mathrm{~cm}^{-1} \mathrm{~s}^{2}$ and $B=2.895 \mathrm{~cm}^{-1} \mathrm{~s}^{2}$ for E7. According to, ${ }^{16}$ we can find the frequency dependence of sound velocity, i.e.

$$
V(\omega)=\left[V_{\infty}^{2}-\frac{V_{\infty}^{2}-V_{0}^{2}}{1+\omega^{2} \tau^{2}}\right]^{1 / 2}
$$

which assumes a single dynamic relaxation time. Here $V_{\infty}$ and $V_{o}$ are high and low frequency limits of velocity and $\tau$ is the relaxation time (i.e. $\tau=1 / \omega$ ). By fitting Figures $1 \mathrm{c}$ and $2 \mathrm{~b}$ with Equation (5), we found $V_{\infty}=1670 \pm 20 \mathrm{~m} / \mathrm{s}$ and $V_{o}=1576 \pm$ $20 \mathrm{~m} / \mathrm{s}$ for ZLI-2861, and $V_{\infty}=1694 \pm 20 \mathrm{~m} / \mathrm{s}$ and $V_{o}=1455 \pm 20 \mathrm{~m} / \mathrm{s}$ for E7. The condition $\omega \tau=1$ is satisfied at the same characteristic frequencies $f_{r}$ which are found from the attenuation fitting. The dispersion of hypersound velocity $\left(V_{\infty}-V_{0}\right) / V_{\infty}$ is positive and is $\sim 6 \%$ for ZLI-2861 and $\sim 14 \%$ for E7.

Now we will consider the relation between hypersonic attenuation anomalies and viscosities of our liquid crystals. The stress tensor requires five viscosity coefficients to describe a compressible nematic. ${ }^{17}$ Different combinations of some coefficients are 
widely used for convenience in describing different properties and in measurements by different methods. For our crystals it is known that one of the Miesowicz viscosity coefficients, $\eta_{2}$ according to, ${ }^{18}$ can be approximated by the bulk viscosity of an unaligned liquid crystal as obtained in an Ostwald-type viscosimeter. The latter is about $20 \mathrm{cP}$ in $\mathrm{ZLI}-2861$ and $39 \mathrm{cP}$ in $\mathrm{E} 7^{19}$ at room temperature in the nematic phase.

Consideration of refractive index anisotropy is essential for correct calculations of $V$ and $\alpha / f^{2}$. Since the optical anisotropy or birefringence $\Delta n\left(=n_{e}-n_{o}\right)$ is small $\left(\Delta n / n_{e} \sim 7.7 \%\right.$ for ZLI-2861 and $12.7 \%$ for E7), we use the average of refractive indexes [i.e. $n_{\text {ave }} \sim\left(n_{e}+n_{o}\right) / 2$ ] to calculate sound velocity and attenuation coefficients. Here $n_{e}$ and $n_{o}$ are extraordinary and ordinary refractive indexes, respectively. ${ }^{19}$ Sound velocity and attenuation anisotropy in the nematic phase is a fundamental property of liquid crystals. ${ }^{17}$ As mentioned above, the anisotropy together with convection (macroscopic time variation in director orientation) probably affect the velocity and $\alpha / f^{2}$ values in our measurements and result in some spread of the points in Figures 1 and 2. The behavior of $\alpha / f^{2}$ in another liquid crystal (CBOOA) ${ }^{20}$ exhibits a characteristic relaxation structure at high ultrasonic frequencies so that the three similar curves correspond to the different sound propagation directions in magnetically aligned crystals. These three similar curves show that the anisotropy is a relatively small effect in comparison with the relaxor behavior of $\alpha / f^{2}$. Therefore we can expect to find qualitative but indubitable evidence of relaxation behavior of $\alpha / f^{2}$ in our disordered samples. We also suppose that in our case one can connect the value of $\alpha / f^{2}$ with an averaged sum $\left(\eta_{\text {ave }}\right)$ of combined bulk $\left(v^{\prime}\right)$ and shear $(v)$ viscosities according to a formula for isotropic liquids in the hydrodynamic theory ${ }^{15}$

$$
\frac{\alpha}{f^{2}}=\frac{2 \pi^{2} \eta_{\text {ave }}}{\rho V_{o}^{3}}=\frac{2 \pi^{2}\left(\frac{4 v}{3}+v^{\prime}\right)}{\rho V_{o}^{3}}
$$

where $\rho$ is the liquid crystal density and $V_{o}$ is the sound velocity in the low-frequency limit if there is a dispersion process as in Figures $1 \mathrm{c}$ and $2 \mathrm{~b}$. The viscosity values calculated by using Equation (6) are shown in Figures $1 \mathrm{~b}$ and $2 \mathrm{a}$ on the right-hand scale.

We mentioned above that a relaxation process at Brillouin frequencies was also found in $\mathrm{MBBA}^{9}$ by comparing the Brillouin linewidths from three different experiments: Steger and Litster $(\mathrm{SL})^{3}$ at $446 \mathrm{MHz}$, Harada and Crooker $(\mathrm{HC})^{9}$ at $2.7 \mathrm{GHz}$ and Clark and Liao (CL) 5 at $7.1 \mathrm{GHz}$ (see Figure 2c). The dispersion "wave" in $\alpha / f^{2}$ corresponds to a reduction in the viscosity value. Data analysis as suggested by Equations (4) and (6) gives for ZLI-2861, E7 and MBBA a series of close values: 30, 56.7 and $26 \mathrm{cP}$. However, we find a qualitative difference between MBBA and our crystals, ZLI-2861 and E7. MBBA possesses another relaxation process at ultrasonic frequencies [8-10] with $f_{r} \sim 13 \mathrm{MHz}$. Equation (4) was used for the sound attenuation analysis with $B$ considered to be independent of frequency. The high-frequency ultrasonic value for $\alpha / f^{2}$ corresponds to the SL point in Figure 2(c). The sound attenuation in this frequency region was found to be of the same magnitude as the reasonable contribution of the bulk and combined shear-bulk static viscosities. ${ }^{8}$ Moreover, the attenuation in the vicinity of the isotropic-nematic phase transition increases in a manner suggested 
by the temperature dependence of the shear viscosity. ${ }^{8}$ High frequency Brillouin measurements in MBBA showed a temperature dependence for $B$ of a similar relaxation type. The solid line in Figure $2 \mathrm{c}$ fits the viscosity reduction suggested to appear due to elimination of substantial amounts of both the shear and bulk viscosities ${ }^{9}$ or due to a thermal relaxation of the vibrational specific heat. ${ }^{5}$

The magnitude of the viscosity reduction shown in Figures $1(\mathrm{~b})$ and 2(a) is proportional to the static values ${ }^{19}$ of the bulk viscosity in ZLI-2861 (20 cP) and E7 (39 cP). In both cases the E7 values are about twice as large as the ZLI-2861 values. Also we can expect a smaller relaxation frequency in a liquid with greater viscosity $\left(f_{r}=1.2 \mathrm{GHz}\right.$ in E7 and $f_{r}=3.2 \mathrm{GHz}$ in ZLI-2861). However, the drastic increase of the static bulk viscosity in the nematic phase of ZLI-2861 from 20 to $2730 \mathrm{cP}$ upon lowering temperature by about $60 \mathrm{~K}^{18}$ does not correlate obviously with the temperature behavior of $\alpha / f^{2}$ from Brillouin scattering. At least, the preliminary results on temperature behavior of the Brillouin components for $\theta=52.5^{\circ}$ in the same region of the nematic phase showed almost no change in linewidth. The Brillouin shift increased from 4.4 to $5.6 \mathrm{GHz}$ so that experiment corresponded to nearly the middle part of the dispersion curve in Figure $1 \mathrm{~b}$ and displayed a slow decrease of $\alpha / f^{2}$.

The behavior of shear viscosities in both ZLI-2861 and E7 seems to be not available now though one can expect an increase for these values also while the temperature decreases in the nematic phase. Accordingly, we have insufficient data to connect the high-frequency relaxation in the nematic phases of ZLI-2861 and E7 with some definite mechanism of viscosity. Probably these relaxations do not play a significant role in the nematic phases. Further investigations at small angles of light scattering and variable temperature would be helpful. Also, time-domain reflectometry which was performed on other liquid crystals to cover the $300 \mathrm{kHz}$ to $10 \mathrm{GHz}$ frequency range, ${ }^{21}$ could prove useful in studying these crystals.

\section{CONCLUSIONS}

We have demonstrated the utility of Brillouin scattering measurements for studying the hypersonic properties of liquid crystals. A variety of different scattering angles from backscattering to nearly forward-scattering provides over a decade of frequency change for most usual fluids. More careful measurements at the smallest angles can extend the frequency region. This region seems to be important for liquid crystal study because of internal relaxation processes in this region. The data for two complex nematic mixtures, E. Merck ZLI-2861 and E7, clearly indicate relaxation anomalies for both hypersonic velocity and attenuation. The existence of a characteristic relaxation process at high frequencies seems to be a common phenomenon for liquid crystals. An analogous behavior appears to be revealed in the classical crystal MBBA. ${ }^{9}$ The MBBA results were not obtained in a set of Brillouin scattering measurements on one sample as we did, but from three different experiments.

The authors would like to express sincere thanks to Prof. George F. Tuthill for helpful discussions. This work was supported in part by DOE Grant DE-FG0687ER45292 and an NRC-CAST Grant. 


\section{References}

1. G. Durand and D. V. G. L. Narasimha Rao, Phys. Lett., 27A, 455 (1968).

2. H. Rosen and Y. R. Shen, Mol. Cryst. Liq. Cryst., 18, 285 (1972).

3. T. Steger and J. D. Litster, In Liquid Crystals and Ordered Fluids, J. F. Johnson and R. S. Porter, Eds., (Plenum Press, New York, 1974).

4. M. Copic and B. B. Lavrencic, J. de Physique 36, C1-89 (1975).

5. N. A. Clark and Y. Liao, J. Chem. Phys., 63, 4133 (1975).

6. I. M. Arefev, V. N. Birukov, V. A. Gladkii, S. V. Krivokhizha, I. L. Fabelinsky and I. G. Chistyakov, $Z h$. Exp. Teor. Fiz. 63, 1729 (1972) [Sov. Phys. JETP 36, 913 (1973)].

7. P. Martinoty and S. Candau, C. R. Acad. Sci. Paris 271, Serie B, 107 (1970).

8. D. Eden, C. W. Garland and R. C. Williamson, J. Chem. Phys., 58, 861 (1973).

9. T. Harada and P. P. Crooker. Mol. Cryst. Liq. Cryst., 42, 283 (1977).

10. S. Candau, P. Martinoty and R. Zana, J. de Physique Lett., 86, L-13 (1975).

11. J. Pelous and R. Vacher, Solid State Comm., 16, 279 (1975).

12. J. M. Vaughan, The Fabry-Perot Interferometer (Adam Hilger, 1989).

13. Zhimin Liu, Ph.D. Thesis, Montana State University (1990).

14. T. Hikita, J. Phys. Soc. Jpn., 53, 1513 (1984).

15. A. B. Bhatia, Ultrasonic Absorption (Clarendon Press, Oxford, 1967).

16. T. Yagi, M. Tokunaga and I. Tatsuzaki, J. Phys. Soc. Jpn., 40, 1659 (1976).

17. P. G. de Gennes, The Physics of Liquid Crystals (Clarendon Press, Oxford, 1974).

18. H. Pruecher, R. Jubb and U. Finkenzeller, The Merck Group Liq. Crys. Newsletter 8, 26 (1991).

19. Preliminary Data Sheet for E7 and ZLI-2861, Advanced Chem. Division, E. Merck Industries.

20. F. Kiry and P. Martinoty, J. de Physique 37, C3-113 (1976).

21. R. Nozakic, T. K. Bose and S. Yagihara, Phys. Rev., A 46, 7733 (1992). 